\title{
A TASK-ANALYSIS-BASED EVALUATION OF SONIFICATION DESIGNS FOR TWO sEMG TASKS
}

\author{
S. Camille Peres
}

\author{
Texas A\&M University, \\ Dept. of Environmental and Occupational Health, \\ Research on the Interface between Humans \\ and Machines Laboratory \\ peres@tamu.edu
}

\begin{abstract}
This paper presents a brief description of surface electromyography (sEMG), what it can be used for, as well as some of the problems associated with visual displays of sEMG data. Sonifications of sEMG data have shown potential for certain applications in data monitoring and movement training, however there are still challenges related to the design of these sonifications that need to be addressed. Our previous research has shown that different sonification designs resulted in better listener performance for different sEMG evaluation tasks (e.g. identifying muscle activation time vs. muscle exertion level). Based on this finding, we speculated that sonifications may benefit from being designed to be task-specific, and that integrating a task analysis into the sonification design process may help sonification designers identify intuitive and meaningful sonification designs. This paper presents a brief introduction to what a task analysis is, provides an example of how a task analysis can be used to inform sonification design, and outlines future research into a task-analysis-based approach to sonification design.
\end{abstract}

\section{INTRODUCTION}

Surface electromyography (sEMG) is a technique for measuring muscle activation onset, muscle activation duration, and muscle exertion level. It is commonly used by researchers [1,2] and physical therapists [3] as a tool for biofeedback [4], as an index of muscle fatigue [2], as a strength training tool [5], and as a motor learning and rehabilitation tool [6].

Typically, EMG data are displayed visually on a computer screen, and while this display modality can work well for certain applications, it has its limitations. Visual displays force EMG technicians to focus their visual attention on a screen, which limits their mobility and prevents them from focusing on the movements of the subject [7]. Additionally, in sports applications, visual displays of EMG data can overload an athlete's visual capacities while the athlete is learning a particular movement [8]. To address these limitations of visual displays of EMG data, researchers have begun exploring auditory displays of EMG data, primarily in the form of parameter-mapping sonifications. Researchers have found that EMG sonifications show potential for

(c) (1) (5) This work is licensed under Creative Commons Attribution - Non Commercial 4.0 International License.

The full terms of the License are available at

http://creativecommons.org/licenses/by-nc/4.0/

\section{Daniel Verona}

\author{
Texas A\&M University, \\ Dept. of Biomedical Engineering, \\ Research on the Interface Between Humans \\ and Machines Laboratory \\ daniel.j.verona@gmail.com
}

improving athletic and exercise performance [8] and identifying musculoskeletal disorders [9]. Additionally, sonification of upper extremity movement within a 3-D space has shown potential for improving motor rehabilitation therapies for stroke patients [10].

These findings suggest that sonification has the potential to be an excellent display modality. Sound is intuitive, the human hearing system has excellent temporal and frequency resolution [7], auditory displays do not restrict a researcher to a computer screen, and sound can have a communal aspect as well - that is to say, an auditory display can afford everyone in a room immediate and simultaneous access to the display (a luxury not common to visual displays). However, despite these advantages, sonification presents its own unique challenges - chief among them is the challenge of display design.

Designing sonifications and choosing mappings that are intuitive, meaningful, and listenable is difficult; thus many different parameters of sound (pitch, loudness, tempo, attack time, spatial location, tremolo, harmonic content, etc.) have been evaluated for use in sonification [11, 12, 13]. Despite this research however, there has been a lack of empirical evaluation and comparison of different sonification designs [14].

We have begun to address this in a recent study (Peres et al., under review) by empirically comparing listener performance between six different sonification designs for sEMG data. We found that different sonification designs resulted in better listener performance for two different sEMG evaluation tasks (results from this study are discussed in further detail below in Section 3). These results indicated that different sonification designs may be better suited to different tasks, which led us to speculate that:

1. Task-specific sonification designs may be helpful in creating effective and meaningful sonifications

2. Basing sonification design on a task analysis (a design tool used in Human Factors in HCI) may help sonification designers identify effective and meaningful mappings for a given task

Applying a task analysis to sonification design is not a new concept [15], however it does not seem to be a wellrepresented approach to sonification design. In order to better understand the effects that a task analysis could have on sonification design and listener performance, we believe it would be beneficial to continue performing empirical comparisons of different sonification designs by comparing task-analysis-based designs (i.e. designs that are tailored to a specific task or function) to classic parameter-based designs that are typically task-agnostic (i.e. designs that map a data 
set to some set of auditory parameters and are not tailored to a specific task or function).

This paper offers a brief description of what a task analysis is, describes two previous task analyses that we performed for the two sEMG evaluation tasks used in our previous study, and outlines future research into the effects of task-analysis-based sonification design on listener performance.

\section{TASK ANALYSIS}

As previously mentioned, a task analysis is an analysis and design tool used in fields such as Human Factors and HCI $[16,17]$. The role of a task analysis can be viewed in several ways, ranging from the entire front-end predesign process, to one element of the front end process, to a range of techniques that come into play at different times during design and development [18]. Common to each of these perspectives however is that a task analysis is meant to provide knowledge about the users, their goals in accomplishing the task, their environment, the manual elements of the task, the cognitive elements of the task, the tools used to perform the task, the duration, order, and complexity of the task, as well as any other unique factors pertaining to the task [19]. Task analysis methods were developed primarily as a means for assessing and reducing human error, though the use of these methods has expanded over time [20].

There are many different types of task analysis methods available and one simple way to categorize them is to divide them into action oriented methods and cognitive methods [21]. Action oriented methods (such as the commonly used hierarchical task analysis) focus on observable actions, or identifying, in top down fashion, the goal of the task, as well as the various subtasks and conditions under which those subtasks must be performed in order to achieve the goal. Cognitive methods, on the other hand, focus on analyzing and outlining the unseen mental processes - diagnosis, decision making, problem solving, etc. - that can give rise to human error [21].

Listening to sonifications for the purpose of data monitoring, analysis, or exploration requires that the listener be able to identify certain characteristics of the sounds that are heard and relate those characteristics to various features of the data in order to make judgments about the data. Since this is primarily a cognitive task (not manual), we advocate using cognitive task analysis methods as tools to understand the listener's task. Section 3 below provides an example of how this can be done and what can be learned from applying this tool to sonification design.

\section{TASK ANLAYSIS OF TWO SEMG EVALUAITON TASKS}

As previously mentioned, we found in our prior research that different sonification mappings resulted in better listener performance for different tasks. In this study, each participant listened to sonifications of two simultaneous channels of sEMG data. One channel was referred to as Muscle A while the other channel was referred to as Muscle B. Each sonification lasted 10 seconds, and in each sonification, both muscles (A and B) started at rest, contracted at close to the same time, and then returned to rest. We asked each participant to identify two characteristics of the muscle activity represented by the sEMG data: which muscle (A or B) activated first and which muscle (A or B) exhibited a higher exertion level.
For the parameter mapping, the sEMG data being sonified were sampled at $1000 \mathrm{~Hz}$, and then averaged into blocks of 100 data points each, creating 10 averaged data values for each second of sEMG data. These averaged values were then played back using SuperCollider's Triangle wave oscillator at a rate of 10 tones per second to preserve the original timing of the sEMG data (e.g. 10 seconds of sEMG data yielded a 10 second sonification). The Pitch, Loudness, and Attack Time of each tone were mapped to the averaged sEMG data values. Pitch was mapped to a range of $200-768$ $\mathrm{Hz}$ (roughly G3 to G5), such that Pitch increased as sEMG data values increased. Loudness was mapped to a range of 50 - $68 \mathrm{~dB}(\mathrm{Z})$, as measured by the SoundMeter app developed for iOS by FaberAcoustical, such that Loudness increased as sEMG data values increased. The Attack Time of each tone was mapped to a range of $0-39 \mathrm{~ms}$, such that Attack Time decreased as sEMG data values increased (i.e. the Attack Time of each tone was longest during muscle relaxation and the Attack Time for each tone became progressively shorter as sEMG data values increased).

Three of the six designs tested were spatialized, meaning that Muscle A was played in the left audio channel and Muscle B was played in the right audio channel. The other three designs were not spatialized, meaning that both Muscles $\mathrm{A}$ and $\mathrm{B}$ were played equally in the left and right audio channels (in the center of the stereo field).

We found that, out of the six designs tested, a Pitch/Loudness with spatialization mapping (PL) resulted in the best listener performance for the task of identifying which muscle contracted first. For the task of identifying which muscle exhibited a higher exertion level, we found that a Pitch/Loudness/Attack Time with spatialization mapping (PLA) resulted in the best listener performance. The nonspatialized mappings showed very poor listener performance.

To understand why these different sonification designs resulted in better listener performance for different tasks, we performed a task analysis for both sEMG data evaluation tasks, and we use these below to offer a possible explanation for the observed performance differences.

\subsection{Task Analysis \#1 - Identifying Which Muscle Contracts First}

Goal: To accomplish this task, the listener must be able to:

1. Understand that the task has started

2. Identify, as quickly and accurately as possible, when each muscle changes from a state of rest to a state of activation

3. Compare the two moments of muscle activation onset

4. Quickly and accurately report which muscle activated first

Sonic characteristics that may facilitate this:

- A distinct and temporally accurate contrast between the sound of a muscle at rest and the sound of muscle activation onset

- A distinct contrast in sound could be facilitated by a change in a number of different sound parameters including pitch, loudness, harmonic content, and timbre

- Temporal accuracy requires that the sonification present the change in muscle state (from rest to activation) at the precise moment which it happens 
Observation: Out of the six designs, the PL mapping resulted in the best listener performance for the task of identifying which muscle contracted first. This mapping used tones with a very short attack time and a fast decay, as depicted in the amplitude envelope diagram shown below in Figure 1:

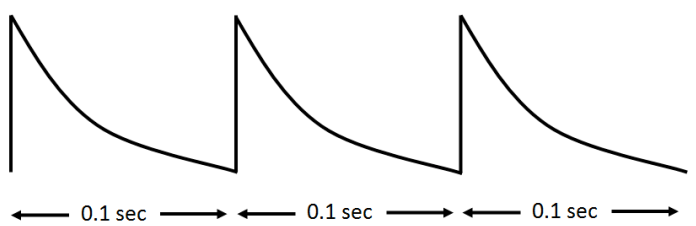

Figure 1 - Shape of the amplitude envelope for three tones in the PL sonification mapping (playing 10 tones per second)

As a result of this amplitude envelope, muscle activation onset was perceived as soon as the tone representing muscle activation onset played. This was not the case in the mappings that incorporated attack time as a parameter (the PLA mapping). For the PLA mapping, the amplitude envelope for each tone at the moment of muscle activation onset looked like that shown below in Figure 2:

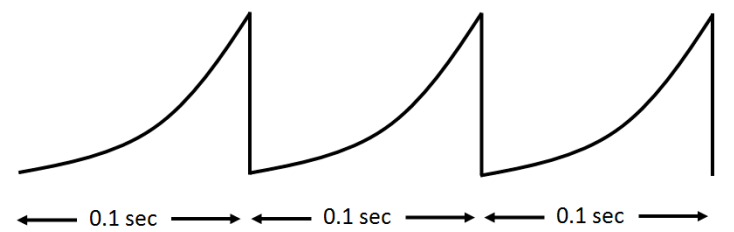

Figure 2 - Amplitude envelope for three tones in the PLA sonification mapping at the point of muscle activation onset

Based on Figure 2, it is clear that, in the PLA mapping, the tone which represented the point of muscle activation onset was not immediately heard by the listener. Thus, while the mappings that included attack time resulted in greater timbral variety (going from a smooth sound while the muscle was at rest to a more percussive sound during muscle activation), this timbral variety may have come at the expense of some temporal accuracy. With the PL mapping being the most temporally accurate out of the six designs, it resulted in the best performance for the task which required temporal accuracy in the sonification (comparing muscle activation times).

\subsection{Task Analysis \#2 - Identifying Which Muscle Exhibits a Higher Exertion}

Goal: To accomplish this task, the listener must be able to:

1. Understand that the task has started

2. Identify when each muscle changes from a state of rest to a state of activation

3. Monitor the level of exertion for each muscle during the muscle activation state

4. Identify when each muscle reverts from a state of activation back to a state of rest

5. Compare the exertion levels for both muscles once the muscle activation state is complete

6. Quickly and accurately report which muscle exhibited a higher exertion level
Sonic characteristics that may facilitate this:

- Easily identifiable sonic differences between the sound of a muscle at rest and the sound of a muscle during contraction

- Easily identifiable sonic differences between various levels of muscle exertion

Observation: Out of the six designs tested, the PLA mapping resulted in the best listener performance for the task of identifying which muscle exhibited a higher exertion level. As discussed previously, including attack time as a parameter of sound in the sonification mapping resulted in greater timbral variety in the sonification (at the expense of some temporal accuracy). With this added timbral variety, the tones in the PLA design sounded smooth and connected while the muscle was at rest, but sounded progressively more percussive and distinct as muscle exertion increased. This not only made it simple to distinguish between the sound of a muscle at rest and the sound of muscle contraction, but also made it easier to distinguish between different levels of muscle exertion, helping to facilitate performance of Step 3 in the above task analysis. The PL mapping (without attack time) did not exhibit this timbral variation between muscle rest and muscle activation, and the listener performance data indicated that this lack impaired the listener's ability to distinguish between various muscle exertion levels. This seems to indicate that variations in timbre, rather than variations in just pitch and loudness, can allow a listener to more easily distinguish between multiple possible data values (in this case multiple different muscle exertion levels).

\subsection{Conclusion}

This task analysis process demonstrates that there can be tradeoffs for using different parameters of sound in sonification mappings. In this case, the tradeoff was between temporal accuracy and timbral variety, as it was not possible to have both at the same time with the designs used. The PL mapping gave better temporal accuracy than the PLA mapping (and the task analysis showed that temporal accuracy was required for the task of identifying which muscle activated first), but the PL mapping did not have the timbral variety of the PLA mapping. This timbral variety helped the listeners better distinguish between relative muscle exertion levels, which resulted in better performance for the task of identifying which muscle had a higher exertion level. We believe that using task analysis methods in this way as a tool for informing sonification design could help sonification designers identify potential tradeoffs in their designs as well as identify potentially useful and meaningful sonification designs for specific tasks.

\section{TASK ANALYSIS IN FUTURE RESEARCH}

In order to better understand the role that a task analysis could play in sonification design, we are investigating the space of task-analysis-based sonification design in work that is currently in the planning stages. We will be performing empirical comparisons between task-analysis-based sonification designs and classic parameter-based designs, which are typically task-agnostic. In order to perform an unbiased comparison of these different sonification mappings, it is crucial to ensure that each task-agnostic mapping used faithfully represents the data in the best way that that particular mapping is capable of. It would be easy to create a 
poor task-agnostic mapping and compare it to a task-analysisbased mapping and "discover" that a task-analysis-based mapping results in better listener performance (conveniently confirming our hypothesis that task-analysis-based designs will result in better performance). To ensure that this sort of bias does not creep in, we will base the task-agnostic designs on designs found in the literature that have been used by other researchers. The task-agnostic designs will also be designs that pertain to human movement, since this research focuses on sEMG data sonification. We will then compare these taskagnostic mappings with task-analysis-based mappings to see how each mapping affects listener performance.

To perform these comparisons, we will identify characteristics of the sEMG data for our listeners to identify after listening to each sonification and then record the accuracy of their answers. We will then compare the accuracy of the listeners' responses between sonification designs in order to determine the effects of design on performance and determine whether or not task-analysis-based designs could improve listener performance.

\section{LINKS TO SOUND FILES}

Pitch/Loudness mapping with spatialization (PL): https://soundcloud.com/user-341542684/pitchloudnessmapping-with-spatialization

Pitch/Loudness/Attack Time mapping with spatialization (PLA):

https://soundcloud.com/user-341542684/pitchloudnessattacktime-mapping-with-spatialization

\section{REFERNCES}

[1] Mabrouk \& Kandil, Surface Multi-Purposes Low Power Wireless Electromyography (EMG) System Design, Int. J. Comput. Appl. (2012) 10-16.

[2] De Luca, The Use of Surface Electromyography in Biomechanics, J. Appl. Biomech. 13 (1997) 135-163.

[3] Kang et al, The Effects of Closed Kinetic Chain Exercise using EMG Biofeedback on PFPS Patients Pain and Muscle Functions, Int. J. Biosci. Biotech. (2014) 55-62.

[4] Steele et al, Electromyography as a Biofeedback Tool for Rehabilitating Swallowing Muscle Function, Applications of EMG in Clinical and Sports Medicine (2012) 311-328.

[5] Croce, The Effects of EMG Biofeedback on Strength Acquisition, Biofeedback and Self-Regulation 11.4 (1986) 299-310.
[6] Giggins et al, Biofeedback in Rehabilitation, J. Neuroeng. Rehab. (2013)

[7] Henkelmann, (2007). Improving the Aesthetic Quality of Realtime Motion Data Sonification. (Technical Report CG-2007-4) Bonn, Germany: Universität Bonn.

[8] Sigrist et al, Augmented Visual, Auditory, Haptic, and Multimodal Feedback in Motor Learning: a Review, Psychon. Bull. Rev. 20 (2013) 21-53.

[9] Pauletto \& Hunt, The Sonification of EMG Data, Proceedings of the $12^{\text {th }}$ International Conference on Auditory Display (2006) 152-157.

[10] Sholz et al, Moving with Music for Stroke Rehabilitation: a Sonification Feasability Study, Ann. N.Y. Acad. Sci. 1337 (2015) 69-76.

[11] Anderson \& Sanderson, Sonification Design for Complex Work Domains: Dimensions and Distractors, J. Exp. Psych: Applied 15.3 (2009) 183-198.

[12] Baier et al, Event-Based Sonification of EEG Rhythms in Real Time, Clinical Neurophysiology 118 (2007) 1377-1386.

[13] Dubus \& Bresin, A Systematic Review of Mapping Strategies for the Sonification of Physical Quantities, PLOS ONE 8.12 (2013) 1-28.

[14] Dubus, Evaluation of Four Models for the Sonification of Elite Rowing, J. Multimodal User Interfaces 5 (2012) 143-156.

[15] Barrass, S. (1997). Auditory Information Design. (Doctoral Dissertation)

[16] Phipps et al, Human Factors in Anaesthetic Practice: Insights from a Task Analysis, British Journal of Anaesthesia 100.3 (2008) 333-343.

[17] Van der Veer et al, GTA: Groupware Task Analysis Modeling Complexity, Acta Psychologica, 91.3 (1996) 297-322.

[18] Janice \& Dennis, (2003). Task analysis. In A. J. Julie \& $\mathrm{S}$. Andrew (Eds.), The human-computer interaction handbook (pp. 922-940): L. Erlbaum Associates Inc.

[19] Kirwan \& Ainsworth, (1992). A guide to task analysis. Philadelphia, PA: Taylor \& Francis.

[20] Berecuartia, (2011). Compilation of Task Analysis Methods: Practical Approach of Hierarchical Task Analysis Methods, Cognitive Work Analysis and Goals, Operations, Methods and Selection Rules. (Masters Thesis)

[21] Embrey, (2000). Task Analysis Techniques. Retrieved from

http://www.humanreliability.com/articles/Task\%20Anal ysis\%20Techniques.pdf 